\title{
Properties of Warm Absorbers and Constraints from Their Optical and UV Line Emission
}

\author{
Stefanie Komossa and Henner Fink \\ Max-Planck-Institut für Extraterrestrische Physik, D-85740 Garching, \\ Germany
}

\section{Introduction and Motivation}

We have studied the warm absorbers in several Seyfert galaxies using both PI and archival ROSAT data. The parameters that govern the emissivity of the ionized material, ionization parameter $U$, warm-absorber column density $N_{\text {wa }}$, and the source intrinsic power-law photon index $\Gamma_{\mathbf{x}}$, are determined from $\mathrm{X}$-ray spectral fits. These are then used to predict the expected IR to UV line emission of the ionized material in the individual objects. In particular, the possibility of a warm-absorber origin of one of the known emission-line regions in AGN is assessed. Good 'candidate components' are, e.g., the coronal-line region, and/or the high-ionization component of the NLR, or the component responsible for broad wings in the Balmer lines. All calculations have been carried out using the code CLOUDY (Ferland 1993).

The objects studied are the Seyfert galaxies NGC 4051, NGC 3227, and Mrk 1298, and the narrow-line Seyfert 1s (NLSy1) RX J1239+24 and RX J1225+20. The derived ionization parameters for the absorbers in the three Seyferts span a representative range from low ionization to high ionization and the optical spectra show (a) high-ionization IR and optical coronal lines (NGC 4051), (b) non-variable broad wings in $\mathrm{H} \alpha$ (NGC 3227), and (c) strong Fe II (Mrk 1298). The 2 NLSy1s exhibit particularly steep X-ray spectra to which a warm-absorber description has been successfully applied.

\section{Seyfert Galaxies}

$N G C$ 4051: We find $\Gamma_{\mathbf{x}}=-2.3$ and for the warm absorber $\log N_{\text {wa }}=22.7$ and $\log U=0.4$ (from the X-ray spectral fits), a limit on the density of $n_{\mathrm{H}} \leq$ $3 \times 10^{7} \mathrm{~cm}^{-3}$ (from variability arguments), which translates into a distance from the nucleus of $r \geq 3 \times 10^{16} \mathrm{~cm}$, and a covering factor that can be as large as unity (from emission-line arguments). The maximum predicted absorber-intrinsic $\mathrm{H} \beta$ emission is about $1 / 220$ of the observed $L(\mathrm{H} \beta)$. Rescaling the strongest expected optical emission line results in an intensity ratio of [FeXIV] $\lambda 5303_{\mathrm{wa}} / \mathrm{H} \beta_{\mathrm{obs}} \approx$ 0.01 , which is consistent with the observed upper limit. Due to the low emissivity of the warm gas, no strong UV-EUV emission lines are produced (e.g., Ne VIII $\lambda 774_{\text {wa }} / \mathrm{H} \beta_{\text {obs }} \leq 0.2$, FeXVI $\lambda 343_{\text {wa }} / \mathrm{H} \beta_{\text {obs }} \leq 2.0$ ). Consequently, no known emission-line component in NGC 4051 can be fully identified with the warm absorber.

$N G C$ 3227: We find $\log U \approx-1.0$ and $\log N_{\text {wa }} \approx 21.5$ for $\Gamma_{\mathrm{x}}=-1.9$. The cold column, $N_{\mathrm{H}} \approx 0.55 \times 10^{21} \mathrm{~cm}^{-2}$, is larger than the Galactic value, but not 
as large as implied by line reddening. Mixing dust with the warm gas could supply the reddening without implying a corresponding cold column. Fitting a sequence of (Galactic ISM-like) dusty absorbers, we find $\log U \approx-0.25$ and $\log N_{\text {wa }} \approx 21.8$. Consequences for the location and line emission of the ionized material are:

1. For the dust-free best fit, the density-scaled distance of the warm absorber is $r \approx\left(n_{9.5}\right)^{-0.5} \times 10^{16} \mathrm{~cm}$, which compares to the typical BLR radius of NGC 3227 of $r \approx 4 \times 10^{16} \mathrm{~cm}$ (Winge et al. 1995). The intrinsic luminosity in $\mathrm{H} \beta$ is about $1 / 30$ of the observed broad $\mathrm{H} \beta$ emission. The strongest line is $\mathrm{C} I V \lambda 1549$, with $\mathrm{CIV}_{\text {wa }} / \mathrm{H} \beta_{\text {obs }} \approx 6$.

2. In the case of dust mixed with the warm gas, the density has to be less than about $10^{7} \mathrm{~cm}^{-3}$ to allow dust survival, i.e. $r \geq\left(n_{7}\right)^{-0.5} \times 9 \times 10^{16} \mathrm{~cm}$, which probably places the absorber between BLR and NLR to explain the stronger observed reddening of the broad lines compared to the narrow lines. The overall line emissivity is strongly reduced.

Mrk 1298: We find $\log U \approx-0.3$ and $\log N_{\text {wa }} \approx 22.2$ for fixed $\Gamma_{\mathbf{x}}=-1.9$. The line emission turns out to be negligible, with $L(\mathrm{H} \beta)_{\mathrm{wa}} \approx(1 / 740) L(\mathrm{H} \beta)_{\mathrm{obs}}$, except for $O$ vi $\lambda 1035_{\mathrm{wa}} / \mathrm{H} \beta_{\mathrm{obs}} \lesssim 0.4$. Wang et al. (1996) mention the existence of strong UV absorption lines. Here we find indeed rather large ion column densities and a corresponding equivalent width for $\mathrm{C}$ IV , for example, of $\log W_{\lambda} / \lambda \approx-2.9$ (for $b=60 \mathrm{~km} \mathrm{~s}^{-1}$ ).

\section{Warm Absorbers in Ultrasoft Seyfert Galaxies?}

$R X J 1239+24$ and $R X J 1225+20$ : The 2 NLSy1s exhibit very steep X-ray spectra (formally $\Gamma_{\mathrm{x}}=-3.6$ and -4.3 , respectively, Greiner et al. 1996). A description in terms of warm absorption proves to be successful, albeit not unique due to the poor photon statistics of the data. We find $\log U \approx 0.8$ and $\log N_{\text {wa }} \approx 23.2$ for RX J1225+20, and $\log U \approx-0.1$ and $\log N_{\text {wa }} \approx 22.8$ for RX J1239+24. The absorber-intrinsic $\mathrm{H} \beta$ emission is rather large and corresponds to about $1 / 10$

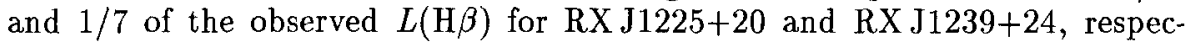
tively. Scaling the predicted [Fe XIV] $\lambda 5303$ emission in order not to conflict with the observed upper limit constrains the covering factor of the gas to $\leq 1 / 6$ in

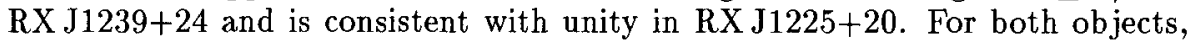
several strong UV emission lines are predicted, e.g., He II $\lambda 1640_{\mathrm{wa}} / \mathrm{H} \beta_{\text {obs }} \approx 1.3$, Fe XXI $\lambda 1345_{\text {wa }} / \mathrm{H} \beta_{\text {obs }} \approx 1.1$, and in the EUV NeVIII $\lambda 774_{\text {wa }} / \mathrm{H} \beta_{\text {obs }} \approx 4.3$ and $\mathrm{MgX} \lambda 615_{\text {wa }} / \mathrm{H} \beta_{\text {obs }} \approx 3.1$ in $\mathrm{RX} \mathrm{J1225+20,} \mathrm{and,} \mathrm{e.g.,} \mathrm{O} \mathrm{vI}_{\mathrm{wa}} / \mathrm{H} \beta_{\text {obs }} \approx 3.5$ in RX J1239+24, assuming the maximum covering consistent with the data.

\section{References}

Ferland, G. J. 1993, University of Kentucky Internal Report.

Greiner, J., et al. 1996, A\&A, 310, 384.

Wang, T., Brinkmann, W., \& Bergeron, J. 1996, A\&A, 309, 81.

Winge, C., et al. 1995, ApJ, 445, 680. 